Supporting Information for the Communication entitled:

\title{
Non-Contact Atomic Force Microscopy of Perfect Single Crystals of Pentacene Prepared by Crystalization from Solution
}

\author{
Kazuya Sato, ${ }^{\dagger}$ Takahiro Sawaguchi, ${ }^{\ddagger}$ Fumihiko Sakata ${ }^{\dagger}$ and Kingo Itaya ${ }^{\dagger, ~} \S$ \\ ${ }^{\dagger}$ Department of Applied Chemistry, Graduate School of Engineering, Tohoku University, 6-6-04 Aoba, Sendai \\ 980-8579, Japan. \\ ${ }^{\ddagger}$ National Institute of Advanced Industrial Science and Technology (AIST), 1-1-1 Higashi, Central 6, Tsukuba, \\ Ibaraki 305-8566, Japan. \\ ${ }^{\S}$ Core Research Evolutional Science and Technology organized by Japan Science and Technology Agency \\ (CREST-JST), Kawaguchi Center Building, 4-1-8 Honcho, Kawaguchi, Saitama 332-0012, Japan. \\ To whom correspondence should be addressed. E-mail: itaya@atom.che.tohoku.ac.jp
}

\section{Stability of Solutions of Pentacene}

In trichlorobenzene, the absorption bands in the UV-visible region disappeared almost totally in air-saturated solution within $20 \mathrm{~min}$, as shown in Figure S1(a). However, pentacene is very stable in the completely degassed solution prepared by the freezing-pumping-thawing cycles. No change in absorption spectra was found for several days or even for months, as shown in Figure S1 (b).

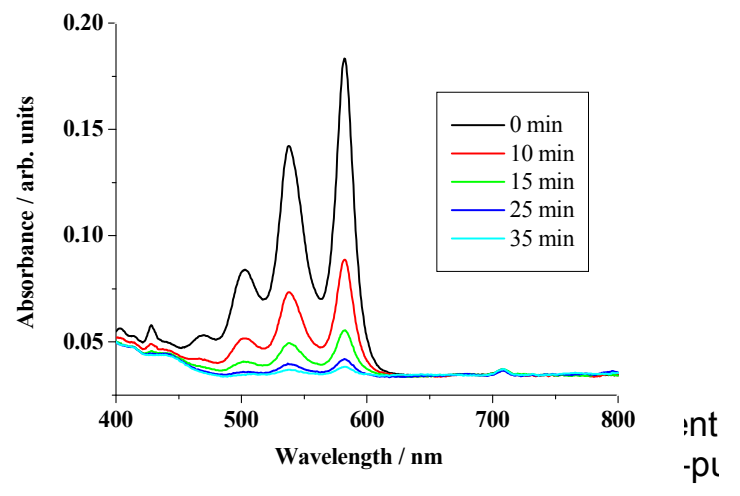

(a)

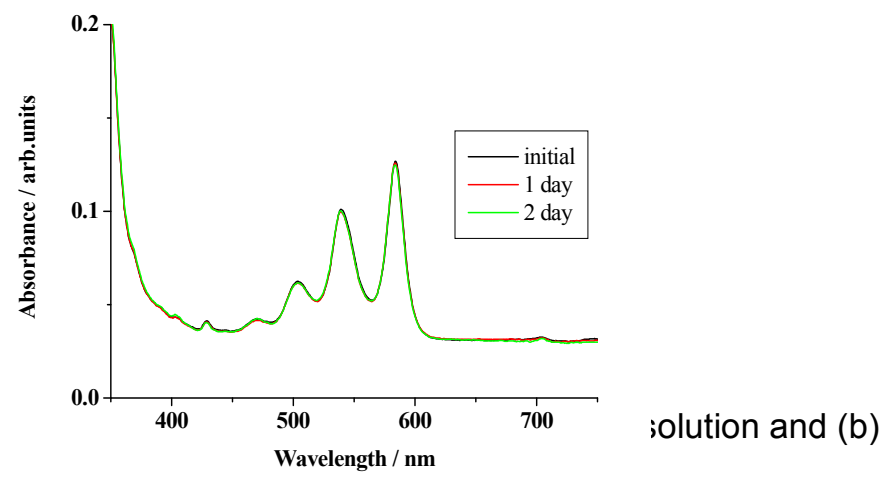

(b) 


\section{Crystallinity of Pentacene}

Micrographs of polarized optical microscope (POM) of single crystals of pentancene are shown in Figure S2. The high crystallinity of single crystals of pentacene grown from the degassed trichlorobenzene solution was confirmed from the images.

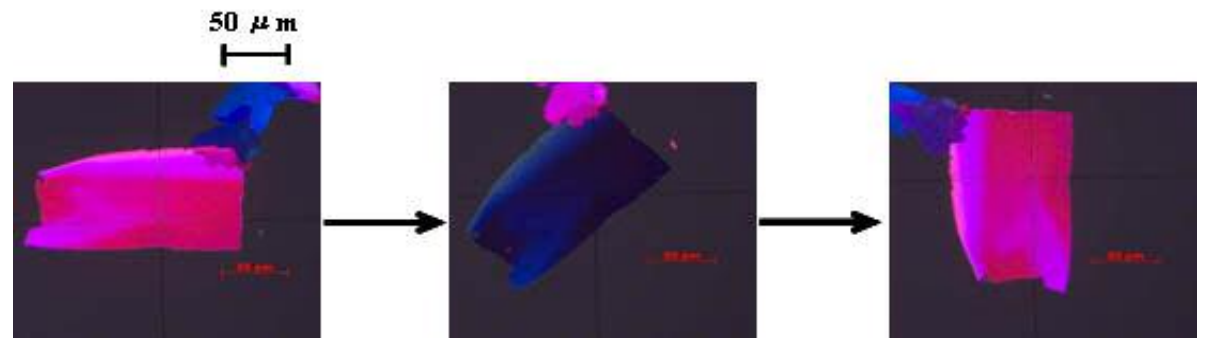

Figure S2. POM micrographs of single crystals of pentancene at $0^{\circ}, 45^{\circ}$, and $90^{\circ}$.

\section{FM-AFM images of Evaporated Pentacene Thin Films}

Typical FM-AFM images of $5 \times 5 \mu \mathrm{m}$ on an evaporated pentacene film is shown in (D). A magnified image $(500 \times 500 \mathrm{~nm})$ is also shown in the inset.

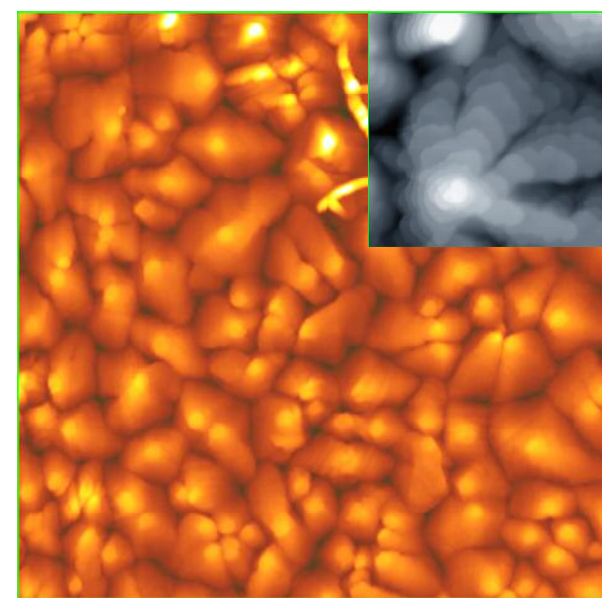

Figure S3. FM-AFM images of the evaporated thin films of pentacene $(5 \times 5 \mu \mathrm{m})$, and the magnified image are also shown in the inset $(500 \times 500 \mathrm{~nm})$. 


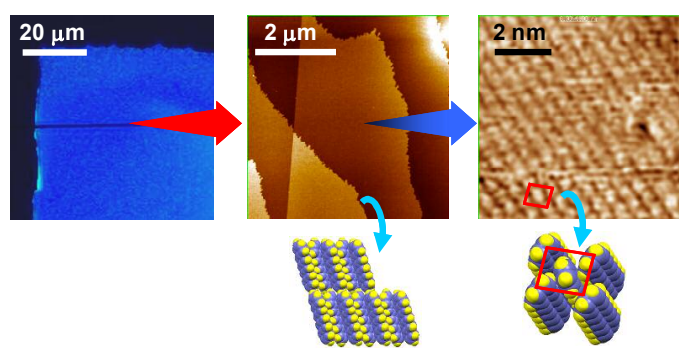

